\section{HIGH QUALITY YET AFFORDABLE}

In addition to the well-known Hogies

Prismatic and Galilean loupes,

Blackwell Supplies is also able to cater to budget-conscious Foundation Dentists with the entry-level Hogies MiniScope flip-up operating loupes.

Together with the Nova frame, Hogies Mini Scope loupes are fully adjustable, thanks to Hogies' patented magnetic system, which can accommodate a range of working heights, angles and inter-pupillary distances. Manufactured from multi-coated glass, Hogies MiniScope loupes are also highly durable and protect the eyes during surgical procedures. Available in $2.5 x$ or $3 \times$ magnification, the capabilities of Hogies MiniScope loupes can further be enhanced by the addition of one or more of the following accessories:

- LED headlight

- Rimless prescription adaptor

- Laser shield

- Polycarbonate protection shield.

Weighing in at a lightweight $48 \mathrm{~g}$, Hogies MiniScope loupes are extremely comfortable to wear and come in a choice of six fashionable colours.

Newly-qualified dental professionals looking for a high quality, yet affordable way to enter the world of surgical magnification need look no further than the Hogies MiniScope, available from Blackwell Supplies.

\section{Reader response number $\mathbf{6 1}$}

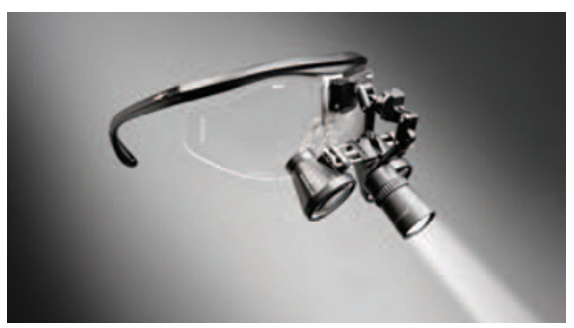

\section{GOLD STAR PERFORMANCE}

Castellini offers both comfort and accuracy with its contemporary range of handpieces to meet the exacting requirements of the dental professional.

The Goldspeed range is manufactured according to the very highest standards of micromechanics, and is the perfect demonstration of more than 70 years of research, design and development from the dedicated team at Castellini. Goldspeed handpieces have a variety of valuable features. For example, integral sanitation is a key attribute as they are fully autoclavable and equipped with a special device which helps to prevent the retraction of contaminants and adheres to HTM 01-05 regulations.

Other convenient features include: high-efficiency intensity fibre optic lighting for long-lasting, white illumination; titanium heads and handgrips for lightness and strength; contra-angled necks for balance and manoeuvrability; they are easily compatible with all ISO 3964 micro-motor couplings; and they are perfectly viable for general, endodontic, restorative, prosthetic and surgical procedures

When a procedure requires accuracy, efficiency and comfort, practitioners require their instruments to perform as well as they do. With the Goldspeed range from Castellini, all elements are considered and combined in a practical, ergonomically sound hand-piece. Excellence is within your grasp. Reader response number 62

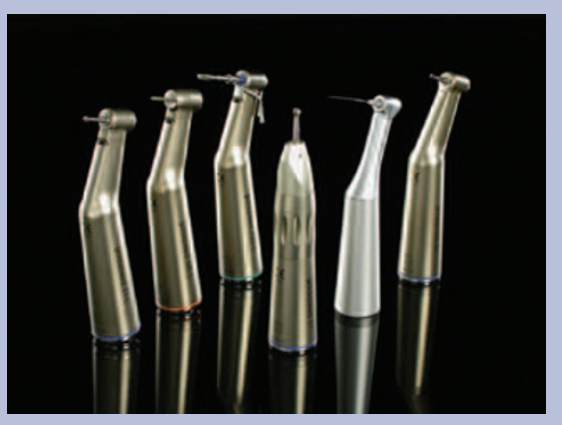

\section{SUPERB INTERPROXIMAL CLEANING}

As an addition to its popular range of interdental brushes, Danish company Tandex has recently launched its new Flexi Max brush: the ultimate interproximal cleaning aid.

The Flexi Max is ergonomically designed with an ultra soft grip handle for ease of use, even for patients with manual dexterity problems and, like every interdental brush in the Tandex range, comes in a range of colour coded sizes, to help patients pick the right brush for them. Suitable for patients of all ages, as well as those with fixed orthodontic appliances, the longer handle and angled head of Flexi Max is ideal. For direct visibility as well as raising compliance and hygiene consistency, the Flexi Max easily fits into the daily oral interdental health care routine of your patients.

The Flexi Max brings patients the same superb design and high quality as other Tandex products, with a little something extra for those patients who find interproximal cleaning difficult. Reader response number 63

\section{WIN AN IPOD TOUCH}

Admor is celebrating the launch of a new dental practice insurance with a prize draw to win a $32 \mathrm{~GB}$ iPod Touch.

To enter the prize draw, simply let Admor know when your practice insurance is up for renewal and allow them to contact you with a quote for their new cover, which is provided and administered by Caunce O'Hara \&t Company Limited.

Or you can visit the Admor website and click on the Dental Practice Insurance button to obtain an online quote and you will automatically be entered in the draw.

The new dental practice insurance includes: accidental damage cover to the contents; protection for your future income; legal expenses; employer's public and products liability; deterioration of drugs; and access to specialist business helplines.

Don't delay; the draw closes on 31 July 2011!

Reader response number 64 